\title{
Selection of chromosomal DNA libraries using a multiplex CRISPR system
}

\author{
Owen W Ryan ${ }^{1 \dagger}$, Jeffrey M Skerker ${ }^{1,2,3}$, Matthew J Maurer ${ }^{1}$, Xin Li $^{1}$, \\ Jordan C Tsai ${ }^{1,4}$, Snigdha Poddar ${ }^{1}$, Michael E Lee ${ }^{1,2}$, Will DeLoache ${ }^{1,2}$, \\ John E Dueber ${ }^{1,2}$, Adam P Arkin ${ }^{1,2,3}$, Jamie HD Cate ${ }^{1,3,4,5 *}$
}

${ }^{1}$ Energy Biosciences Institute, University of California, Berkeley, Berkeley, United States; ${ }^{2}$ Department of Bioengineering, University of California, Berkeley, Berkeley, United States; ${ }^{3}$ Physical Biosciences Division, Lawrence Berkeley National Laboratory, Berkeley, United States; ${ }^{4}$ Department of Molecular and Cell Biology, University of California, Berkeley, Berkeley, United States; ${ }^{5}$ Department of Chemistry, University of California, Berkeley, Berkeley, United States
*For correspondence: jcate@lbl.gov

Present address: ${ }^{\dagger} \mathrm{BP}$ Biofuels Global Technology Center, San Diego, United States

Competing interests: See page 13

Funding: See page 13

Received: 16 June 2014 Accepted: 17 August 2014 Published: 19 August 2014

Reviewing editor: Elisa Izaurralde, Max Planck Institute Development Biology, Germany

(c) Copyright Ryan et al. This article is distributed under the terms of the Creative Commons Attribution License, which permits unrestricted use and redistribution provided that the original author and source are credited.
Abstract The directed evolution of biomolecules to improve or change their activity is central to many engineering and synthetic biology efforts. However, selecting improved variants from gene libraries in living cells requires plasmid expression systems that suffer from variable copy number effects, or the use of complex marker-dependent chromosomal integration strategies. We developed quantitative gene assembly and DNA library insertion into the Saccharomyces cerevisiae genome by optimizing an efficient single-step and marker-free genome editing system using CRISPR-Cas9. With this Multiplex CRISPR (CRISPRm) system, we selected an improved cellobiose utilization pathway in diploid yeast in a single round of mutagenesis and selection, which increased cellobiose fermentation rates by over 10 -fold. Mutations recovered in the best cellodextrin transporters reveal synergy between substrate binding and transporter dynamics, and demonstrate the power of CRISPRm to accelerate selection experiments and discoveries of the molecular determinants that enhance biomolecule function.

DOI: 10.7554/eLife.03703.001

\section{Introduction}

Directed evolution using living systems allows selections for improved biomolecule function to be directly coupled to phenotype. However, present directed evolution systems require the use of extrachromosomal gene libraries encoded in bacteriophage or plasmids, which suffer from high levels of copy number variation (Yokobayashi et al., 2002; Turner, 2009; Esvelt et al., 2011). For example, in the yeast Saccharomyces cerevisiae, common yeast centromere containing (CEN) plasmids vary widely in copy number per cell resulting in highly variable expression levels from cell to cell (Figure 1). Ideally, the selective pressure applied to evolve improved or new biomolecule function should be limited to the DNA sequence level and not to gene copy number in a given cell (Zhou et al., 2012). To overcome copy number variation in yeast, the standard genetic tool of integrating linear DNA into the genome by homologous recombination is too inefficient to insert DNA libraries without multiple steps that rely on dominant selectable markers for chromosomal integrations (Wingler and Cornish, 2011) or specialized strain backgrounds (DiCarlo et al., 2013a).

Bacterial type II CRISPR-Cas9 genome editing has been used successfully in several eukaryotic organisms (Cong et al., 2013; DiCarlo et al., 2013b; Mali et al., 2013a, 2013b; Shalem et al., 2014) but has not been adapted for selection experiments. Genome editing CRISPR-Cas systems require a Cas9 endonuclease that is targeted to specific DNA sequences by a non-coding single guide RNA (sgRNA) (Jinek, et al., 2012; Jinek et al., 2014). The Cas9-sgRNA ribonucleoprotein complex 
eLife digest Over the course of billions of years, natural evolution has produced new proteins and adapted existing ones so that they work better. Scientists have learned how to use the principles that underlie evolution to similarly engineer proteins in the laboratory. This process, known as directed evolution, is a powerful tool for improving how proteins function. Directed evolution normally involves mutating the gene that encodes the protein of interest, selecting the genes that produce the most promising proteins for another round of mutation, and repeating the process until the desired protein function is achieved.

In the first step of directed evolution, a gene is usually mutated randomly in order to create a large 'library' of different forms of the gene. These are joined to circular pieces of DNA known as plasmids that can replicate themselves inside cells. However, the number of plasmids than can be taken up differs from cell to cell. This complicates experiments, and the ideal directed evolution experiment would have the same number of plasmids, or target genes, being delivered into each cell.

Ryan et al. have developed a new method for performing directed evolution experiments that uses a recently developed technique called the CRISPR-Cas9 system. This can make direct changes to a DNA strand such as inserting or deleting specific sequences that code for proteins. Ryan et al. used the CRISPR-Cas9 system to create multiple DNA breaks simultaneously across the genome of yeast cells, and joined 'barcoded' DNA or DNA for intact genes to these breaks. This avoids the need to use plasmids to introduce foreign DNA into cells. Ryan et al. have named this method the Multiplex CRISPR (or CRISPRm) system.

Having established CRISPRm, Ryan et al. tested whether it could be used to engineer improved proteins by attempting to modify a transporter protein called CDT-1. This protein transports the sugar cellobiose into yeast cells, where it can be converted into alcohol by fermentation. This is important for making biofuel from plants. After just one round of directed evolution using CRISPRm, Ryan et al. successfully isolated a form of the CDT-1 protein that increased the rate of fermentation over 10-fold; hence this CDT-1 variant could be used to increase biofuel production.

In the future, it will be important to implement multiple selection rounds with CRISPRm, and to test how large the DNA libraries can be for directed evolution. In time, CRISPRm could find use in evolving and engineering different combinations of genes, metabolic pathways, and possibly entire genomes.

DOI: 10.7554/eLife.03703.002

precisely generates double-strand breaks (DSBs) in eukaryotic genomes at sites specified by a 20-nucleotide guide sequence at the $5^{\prime}$ end of the sgRNA that base pairs with the protospacer DNA sequence preceding a genomic protospacer adjacent motif (PAM) (Sternberg et al., 2014). The presence of the Cas9-produced DSB in genomic DNA can increase the rate of homology-directed repair (HDR) with linear DNA at the DSB locus by several 1000-fold (Storici et al., 2003; DiCarlo et alo, 2013b) potentially enabling high-throughput experimental methods. CRISPR-Cas9 could therefore be useful in probing industrial $S$. cerevisiae strains, which are more robust compared to laboratory strains (Kerr and Service, 2005; Farrell et al., 2006; Rubin, 2008), but for which few genetic tools are available, due to the fact that these strains are often polyploid with low-efficiency mating and sporulation.

\section{Results}

To optimize the efficiency of HDR in S. cerevisiae mediated by Cas9, we first constructed a plasmid expressing Streptococcus pyogenes Cas9 fused to a nuclear localization signal (Cas9-NLS) that efficiently localizes to the nucleus of yeast cells (Figure 2A,B; Jinek et al., 2012). We used an intermediate strength promoter, $P_{R N R 2}$, to express Cas 9 for genome targeting experiments, because Cas 9 (Cas9-NLS-His8x) expression from the $P_{R N R 2}$ promoter resulted in yeast strains with near wild type fitness whereas Cas9 expressed using strong yeast promoters such as $P_{\mathrm{TDH}}$ (Lee et al., 2013) reduced yeast fitness relative to wild type cells (Figure 2 C). Second, we designed a new sgRNA architecture in order to improve its expression and function. Cellular levels of sgRNAs correlate with the efficiency of Cas9-mediated genome targeting in mammalian cells (Hsu et al., 2013). Controlled expression of nuclear-localized RNAs and the correlation between sgRNA levels and Cas9-editing in yeast has not 


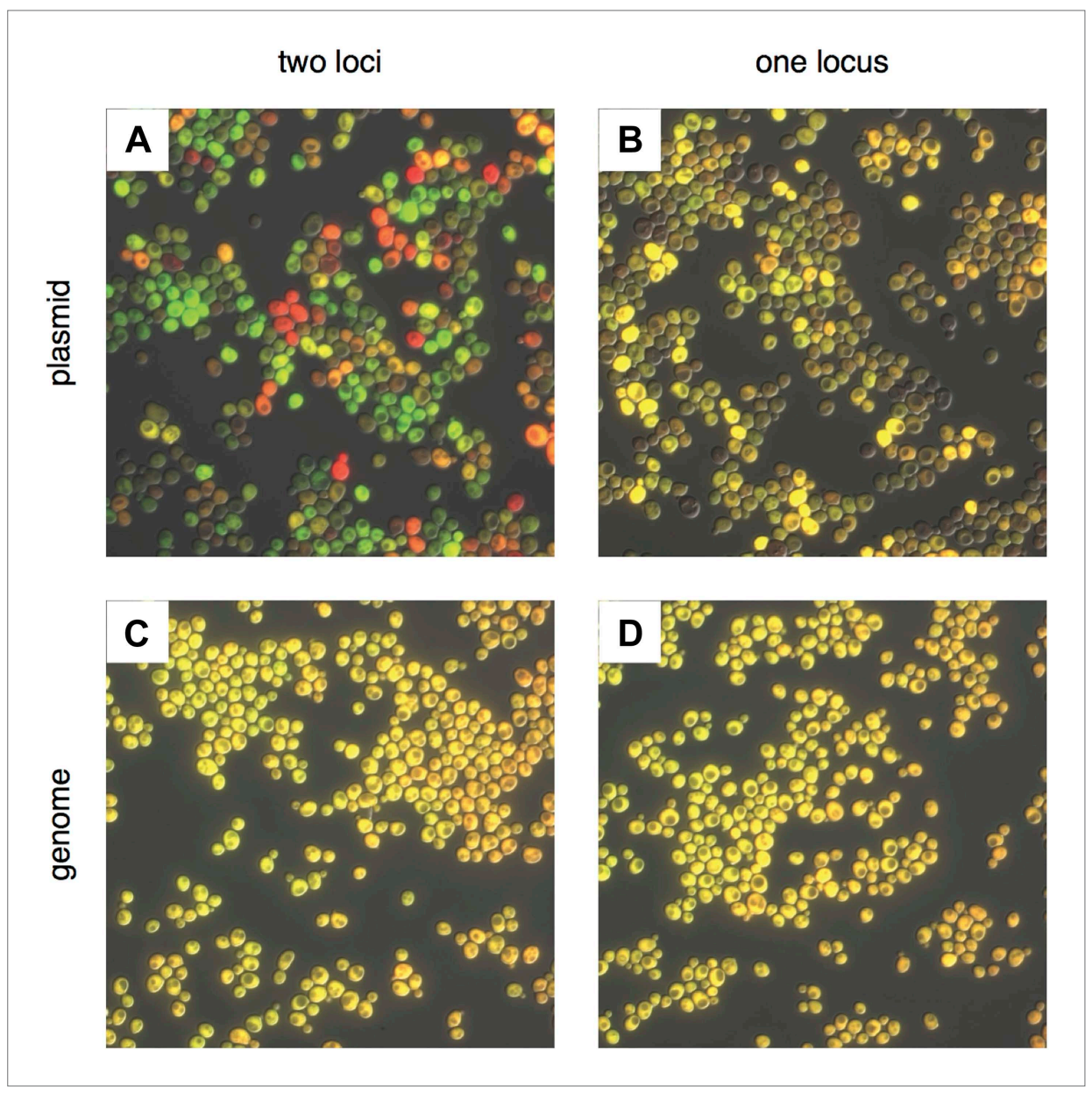

Figure 1. Comparing noise in plasmid and genomic expression of two fluorescent proteins. Proteins mRuby2 (false-colored red) and Venus (false-colored green) were expressed in the same cell with a strong, $P_{T \text { THз }}$ promoter. (A) Fluorescent proteins expressed on two separate CEN plasmids; (B) in tandem on a single CEN plasmid; (C) integrated into two different loci (LEU2 and URA3) in the S. cerevisiae genome; and (D) integrated in tandem at a single locus (URA3) in the genome. Each image was auto-exposed for both red and green channels, with yellow showing co-expression of mRuby 2 and Venus. The variability in the relative expression of the two fluorescent proteins is reduced by moving from two plasmids to one, and the variability in total expression is reduced by moving to the genome. The difference between integrating into one or two loci in the genome is minimal. DOI: 10.7554/eLife.03703.003

been explored. To better control cellular levels of correctly folded sgRNA, we developed a modular design by fusing the sgRNA (Mali et alo, 2013a) to the $3^{\prime}$ end of the self-cleaving hepatitis delta virus (HDV) ribozyme (Ke et alo, 2007; Webb et al., 2009; Figure 2D). We reasoned that the structured ribozyme would protect the $5^{\prime}$ end of the sgRNA from $5^{\prime}$ exonucleases (Houseley and Tollervey, 2009). Additionally, the HDV ribozyme would cleave the RNA immediately $5^{\prime}$ of the ribozyme, removing extraneous RNA sequences and allowing flexibility in which promoters can be used, such as tRNAs whose DNA sequences also serve as RNA polymerase III promoters (Marck et al., 2006). Ribozyme removal of the tRNA would then separate transcription initiation from within the tRNA sequence (Marck et al., 2006) and tRNA nuclear export (Köhler and Hurt, 2007) from the process of forming functional sgRNA complexes with Cas9.

To quantify the efficiency and specificity of HDR with the new Cas9/sgRNA format, phenotype screens were performed by the co-transformation of a single plasmid that expresses the Cas 9 protein and one or more HDV ribozyme-sgRNAs, containing a 20-nucleotide guide sequence that matches a specific site in the yeast genome, along with a linear double-stranded DNA (Figure 2E). The linear dsDNA, which contains a unique 20-mer barcode (Giaever et al., 2002) flanked by common primer sequences and 50 base pairs of DNA homologous to the regions proximal to the PAM motif 
A

$\mathbf{N}$

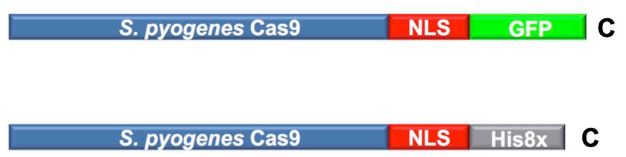

B
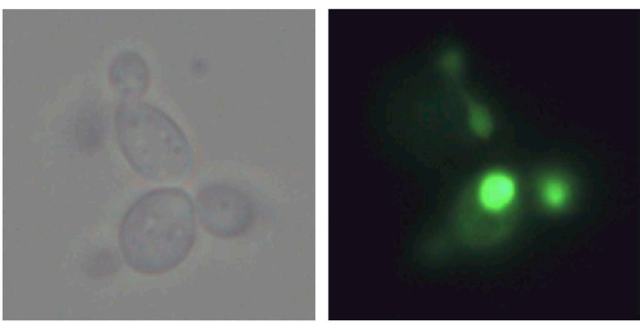

C

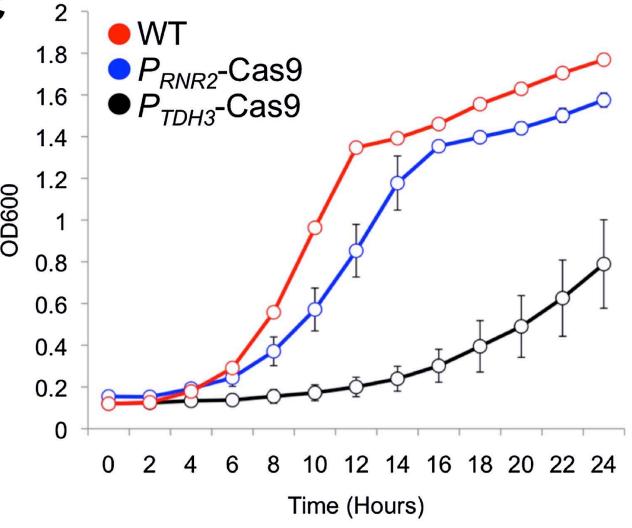

D

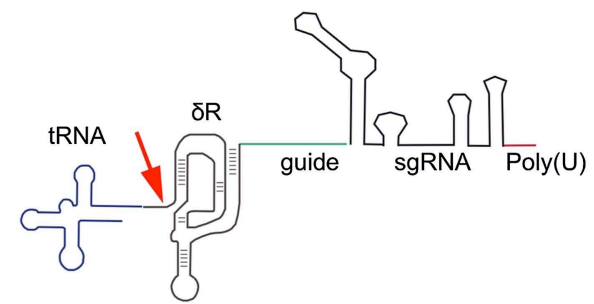

E

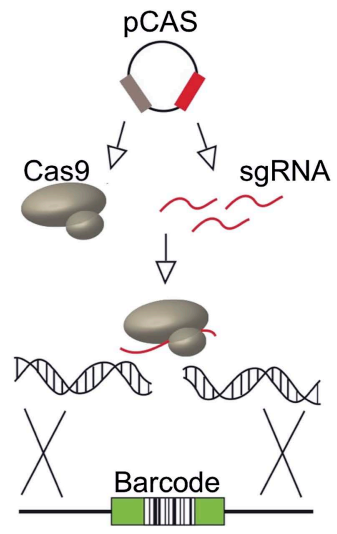

$\mathbf{F}$

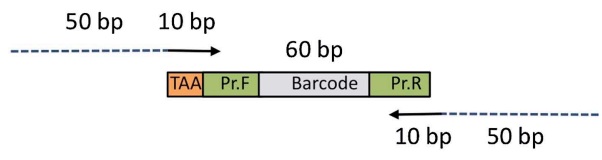

Figure 2. The engineered CRISPRm platform. (A) Cas9 construct used for nuclear localization experiments. The S. pyogenes Cas9 protein was tagged with a C-terminal nuclear localization motif and a green fluorescent protein (GFP) (green). For genome editing experiments, the S. pyogenes Cas9 protein was tagged with a C-terminal nuclear localization motif and a Histidine affinity tag (gray). (B) Cellular localization of Cas9-GFP in exponentially growing S. cerevisiae cells. The Cas9-GFP protein was expressed from the TDH3 promoter in this experiment. Left, bright field image; right fluorescence microscopy. (C) Growth profiles of yeast expressing Cas9 from a strong promoter $P_{T D H 3}$ (black) or a weaker promoter $P_{R N R 2}$ (blue) relative to wild type (red). (D) The mature sgRNA contains a $5^{\prime}$ hepatitis delta virus (HDV) ribozyme ( $\delta R$, brown), 20mer target sequence (green), sgRNA (black) and RNA polymerase III terminator (red). The RNA polymerase III promoter tRNA (blue) is catalytically removed by the HDV ribozyme (red arrow). (E) Schematic of yeast Cas9 targeting. Cas9 and sgRNA are expressed from a single plasmid, form a complex, and cleave targeted genomic DNA, which is repaired using a barcoded oligonucleotide. (F) The linear barcoded repair DNA molecule. Each repair DNA contains a 5' TAA stop codon (gold), a forward primer sequence (green), a unique molecular barcode (gray), and a 3' reverse primer (green) (Giaever et al., 2002). Barcodes are amplified using a forward primer that contains $50 \mathrm{bp}$ of homology (blue) to the genome targeting site and a reverse primer that contains $50 \mathrm{bp}$ of homology to the genome targeting site. The $50 \mathrm{bp}$ of genomic targeting sequence are each $10 \mathrm{bp}$ proximal to the PAM motif, resulting in a $20 \mathrm{nt}$ deletion and barcode oligonucleotide integration.

DOI: 10.7554/eLife.03703.004

(Figure 2F), acts as a template for DNA repair by HDR, resulting in a unique markerless and barcoded insertion allele. With a single sgRNA, we found tRNA sequences used as RNA polymerase III promoters resulted in nearly $100 \%$ efficient barcode insertion in diploid yeast at the URA3 locus, resulting in 5-fluororotic acid resistance (Boeke et al., 1984) whereas the non-tRNA promoters were mostly ineffective (Figure 3A; Supplementary file 1A). We also assessed target sequence bias by targeting 11 unlinked yeast genes in diploid S288C yeast cells using tRNA ${ }^{\mathrm{Tyr}}$ as the RNA polymerase III promoter 


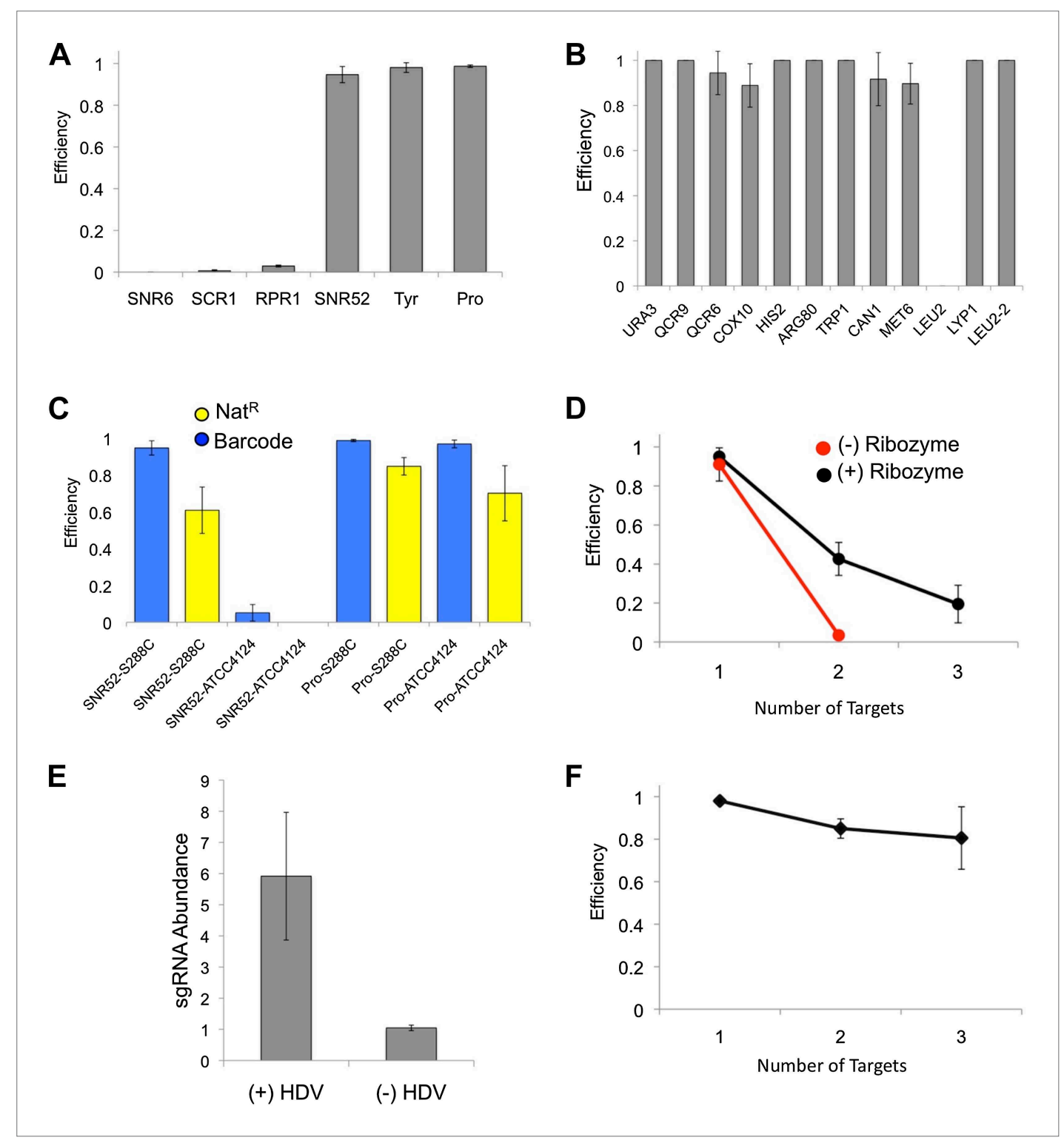

Figure 3. CRISPRm barcode insertion in yeast cells. (A) Targeting efficiency at the URA3 locus in diploid S288C yeast using RNA polymerase III promoters ( $x$ axis) to drive the expression of the sgRNA. (B) Targeting efficiency measured across 11 loci in 10 genes in diploid cells of yeast strain S288C, using the tRNA $A^{\text {Tyr }}$ promoter. (C) Single fragment barcode integration (blue) and three-fragment Nat ${ }^{\mathrm{R}}$ cassette integration (yellow) efficiency in S288C diploid and ATCC4124 polyploid strains. For each experiment the promoter and strain are indicated as promoterstrain (e.g., SNR52-S288C). (D) Efficiency of multiplex insertion of barcoded DNA in diploid yeast cells with the 5' HDV ribozyme (black) and without the 5' HDV ribozyme (red). Triplex targeting without the 5' HDV ribozyme was not tested. The tRNA ${ }^{\text {Tyr }}$ promoter was used in these experiments. (E) The addition of a $5^{\prime}$ HDV ribozyme increases the intracellular levels of sgRNA by sixfold. The RT-qPCR experiments were carried out in biological triplicate, with the mean and standard deviation shown. (F) Efficiency of targeting one (URA3), two (URA3, LYP1) and three (URA3, LYP1 and COX10) in haploid S288C yeast cells. The tRNA ${ }^{\text {Tyr }}$ promoter was used for sgRNA expression. DOI: 10.7554/eLife.03703.005

(Supplementary file 2A). We found that targeting was highly efficient for every genomic target except LEU2, which at first was weak but was restored to $100 \%$ by selecting a different guide sequence (LEU2-2) as the sgRNA (Figure 3B). We also verified that our CRISPR-Cas9/sgRNA system did not result in non-specific genome targeting by sequencing 9 of the targeted strains (Supplementary file 1B). We also tested efficacy of our approach in the polyploid industrial S. cerevisiae strain ATCC4124 that has superior tolerance and productivity phenotypes (Ness et al., 1993). The non-tRNA promoter 
previously used in haploid yeast (DiCarlo et al., 2013b), $P_{\text {SNR52 }}$ functioned in diploid S288C yeast but failed to result in targeting in the polyploid industrial yeast ATCC4124; in contrast, the tRNAPro promoter enabled highly-efficient barcode insertion into all copies of the URA3 locus in ATCC4124, which we term cis-multiplexing, that is introducing a double-stranded DNA break at a single genomic locus across all chromosomes (Figure 3C; Supplementary file 1A). Notably, tRNA $A^{\text {Tyr }}$ was inefficient at targeting in ATCC4124 though it was effective in S288C diploid (Supplementary file 1A), indicating that the choice of tRNA used for the expression of the HDV-sgRNA impacts multiplexing in a straindependent manner.

Since the efficiency of cis-multiplexing with our CRISPR-Cas9/sgRNA system is nearly $100 \%$ using one sgRNA, we next tested its ability for trans-multiplexed CRISPR-Cas9 genome editing, that is introducing double-stranded DNA breaks at multiple genomic loci simultaneously, using tRNAs to drive the expression of the HDV-sgRNAs. We simultaneously targeted two and three unlinked genomic loci in diploid cells for loss-of-function mutagenesis by introducing a single plasmid expressing Cas9 and two or three sgRNAs with two or three gene-specific barcoded DNA molecules, respectively. The efficiency of duplex and triplex targeting (requiring four and six chromosome cuts, respectively) with two sgRNAs (URA3 and LYP1) or three (URA3, LYP1 and COX10) was $43 \%$ and 19\%, respectively (Figure 3D; Supplementary file 1A). This multiplexed efficiency was dependent on the presence of the HDV ribozyme $5^{\prime}$ of the guide sequence as duplex targeting dropped to $3.5 \%$ in cells with sgRNA lacking the $5^{\prime}$ HDV ribozyme (Figure 3D; Supplementary file 1A). We measured the relative cellular abundance of sgRNAs expressed by $P_{S N R 52}$, with and without the $5^{\prime}$ HDV ribozyme, by reverse transcription quantitative polymerase chain reaction (RT-qPCR) and found that the presence of the ribozyme increases the intracellular abundance of the sgRNAs by sixfold (Figure 3E), consistent with the model that sgRNA abundance is rate limiting for CRISPR/Cas targeting (Hsu et alo, 2013). Haploid trans-multiplexing showed a minimal loss in activity scaling from one to three loci (up to three chromosome cuts) (Figure 3F; Supplementary file 1A). These experiments demonstrate that our multiplexed CRISPR/Cas9 system, which we term CRISPRm, is powerful enough to generate multiple marker-free targeted mutations in the yeast genome in a single experiment and that the $5^{\prime}$ tRNA-HDV ribozyme sequence is required for higher order multiplex targeting.

Having established CRISPRm for cis- and trans-multiplex targeting, we next tested its capabilities in engineering genes and pathways integrated in the yeast genome. We first tested whether CRISPRm could be used to assemble foreign genes as chromosomal integrations. We tested the efficiency of in vivo assembly of a functional nourseothricin-resistance $\left(\mathrm{Nat}^{\mathrm{R}}\right)$ gene from three overlapping PCR products encoding a transcription promoter, protein open reading frame (ORF) and transcription terminator at a single locus using cis-multiplexing in the diploid S288C strain. The efficiency of Cas9-mediated integration and assembly of these three DNA fragments to the correct (URA3) locus was measured by a combination of 5 -fluoroorotic acid resistance $\left(5-\mathrm{FOA}^{R}\right)$ and $\mathrm{Nat}^{R}$. We found $85 \%$ efficiency of targeting and assembly in both copies of the URA3 locus in diploid yeast $\mathrm{S} 288 \mathrm{C}$ cells, and $70 \%$ in polyploid ATCC4124 by using the tRNA ${ }^{\text {Pro }}$ sequence as the sgRNA promoter (Figure 3C; Supplementary file 1A), whereas $P_{S N R 52}$-dependent targeting was weaker in S288C cells and non-existent in ATCC4124 cells (Figure 3C; Supplementary file 1A). Thus, CRISPRm enables the one-step markerless assembly of functional genes from multiple fragments into the $S$. cerevisiae genome, resulting in homozygous insertions into both diploid and polyploid yeast strains.

Finally, we tested whether CRISPRm could be used for in vivo selections of improved protein function. We targeted a cellodextrin utilization pathway from the cellulolytic fungus Neurospora crassa, comprised of the cellodextrin transporter (cdt-1) and an intracellular $\beta$-glucosidase (gh1-1), to assemble in vivo in diploid $S$. cerevisiae. In combination, these two genes result in yeast cells capable of consuming the disaccharide cellobiose, which could be used to improve biofuel production (Galazka et al., 2010). To select for improved cellobiose utilizing strains we used error-prone PCR to amplify the cdt-1 gene. We co-transformed the library of mutated cdt-1 alleles, along with overlapping dsDNA fragments for the promoter and terminator, for assembly and integration into the URA 3 locus (ura3:: $P_{P G K 1}-c d t-1-T_{A D H 1}$ library) of a diploid strain with a previously integrated $\beta$-glucosidase gh1-1 gene (lyp 1:: $P_{\left.\mathrm{TDH}_{3}-g h 1-1-T_{\mathrm{CYC}}\right)}$ using CRISPRm. We then grew the transformants in medium containing cellobiose as the sole carbon source to select for functional cdt-1 alleles and identified one strain with 2.6-fold (homozygous cdt-1 N209S/I354N/S360P/T406S/W531L allele) and one strain with 1.7fold (homozygous cdt-1 Q45H/F262Y/F533Y allele) increased cellobiose utilization capacity over wild type cdt-1 (Figure 4A). The mutations were mapped in CDT-1 onto structures of the homologous 
A

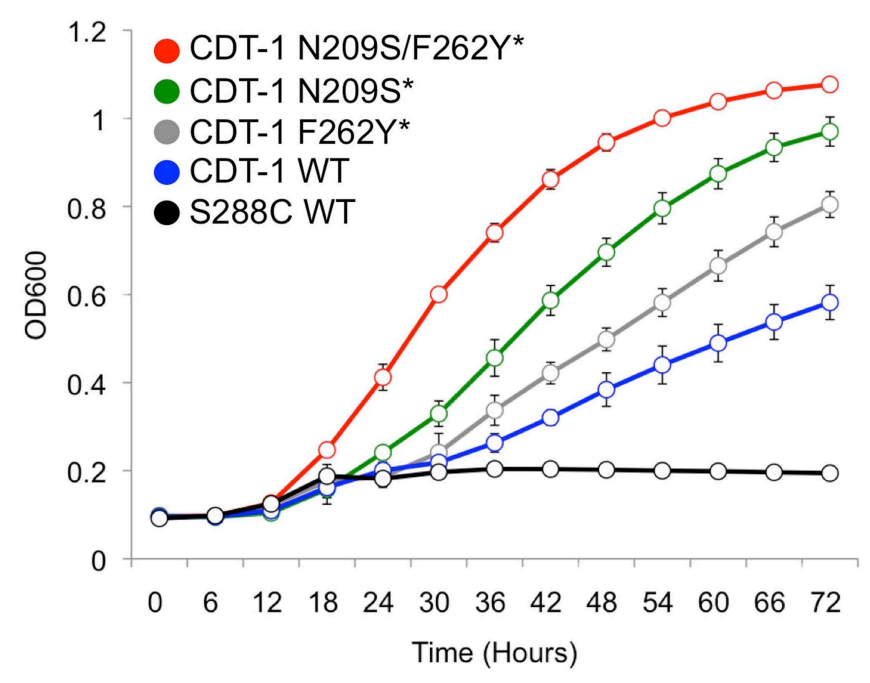

C

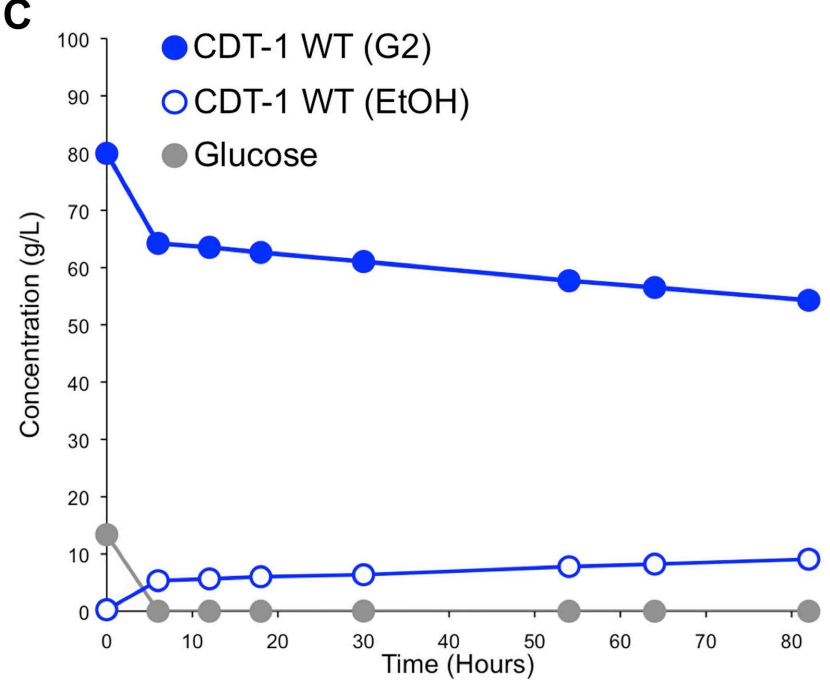

B

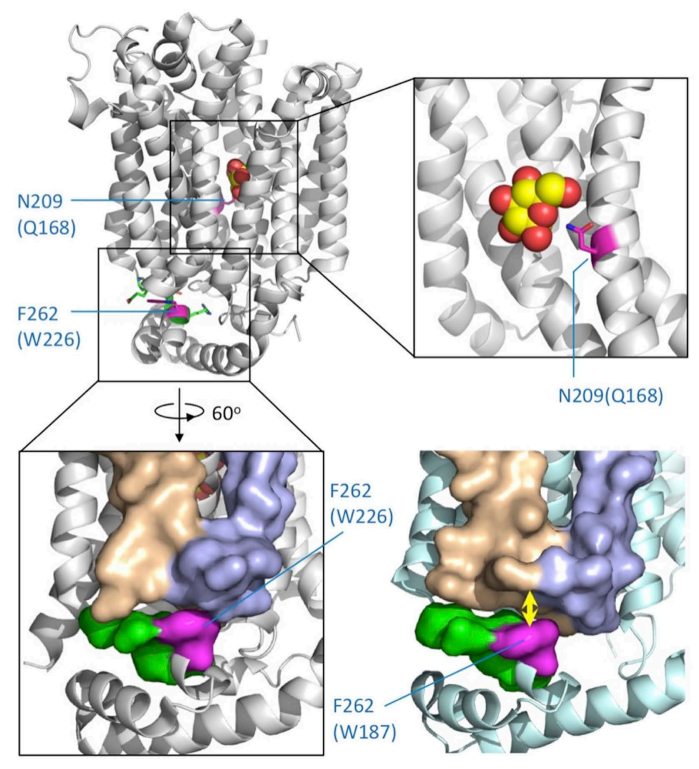

D

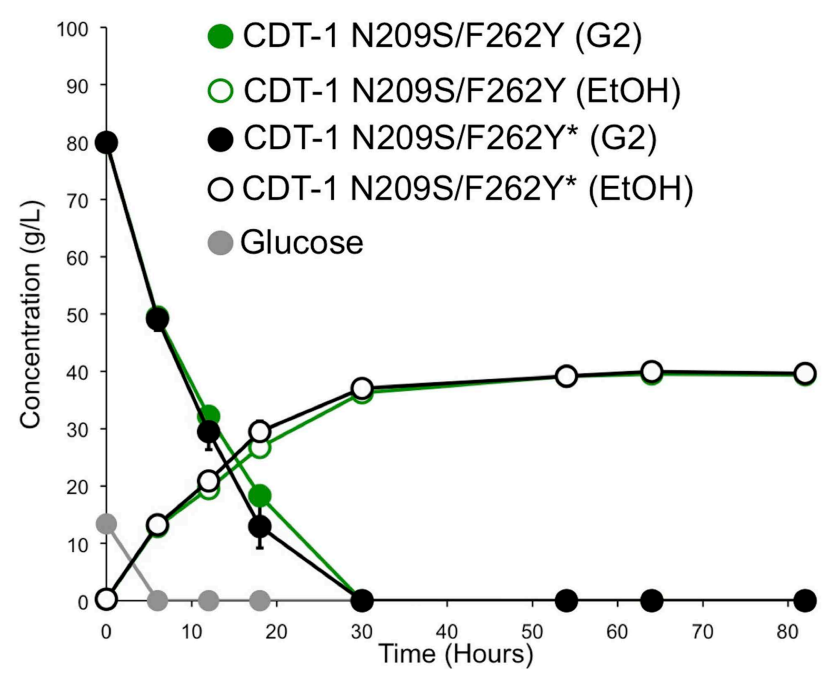

Figure 4. CRISPRm mediated insertion and evolution of chromosomal DNA libraries for in vivo protein engineering. (A). Utilization of cellobiose in

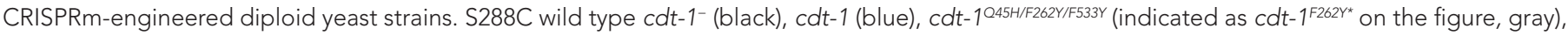

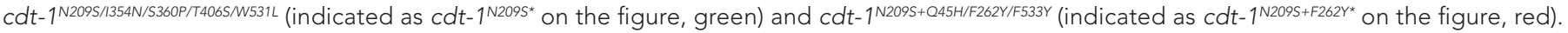
(B) Location of mutations at conserved sites in the evolved CDT-1 transporter structure. The mutated residues are colored magenta in the top figure, and are mapped onto the E. coli XylE transporter in the outward-facing configuration bound to glucose (upper panels and lower left, PDB entry 4GBZ), and onto the S. epidermidis GlcP transporter in the inward-facing configuration (lower right, PDB entry 4LDS). Amino acids in parentheses are the sequences in XylE or GlcP. (C) Fermentation of cellobiose by wild-type cdt-1 expressed from a chromosomally-integrated copy in diploid S288C yeast. Cellobiose is indicated as $\mathrm{G} 2$, ethanol as $\mathrm{EtOH}$. The rate of cellobiose consumption was $0.13 \mathrm{~g} \mathrm{~L}^{-1} \mathrm{hr}^{-1}$. (D) Fermentation of cellobiose by mutant versions of cdt-1 expressed from chromosomally-integrated copies. Glucose consumption (gray) was identical in both strains. The rate of cellobiose consumption for both strains was over $2.0 \mathrm{~g} \mathrm{~L}^{-1} \mathrm{hr}^{-1}$. In (C and D), values and error bars represent the means and standard deviations of three independent biological replicates.

DOI: 10.7554/eLife.03703.006

major facilitator superfamily (MFS) hexose-like transporters XylE from Escherichia coli (Sun et al., 2012) and GlcP from Staphylococcus epidermidis (lancu et al., 2013) (Figure 4B). Notably, one site maps to a site that likely interacts directly with the sugar substrate (N209S) (Figure 4B). Whereas two mutations in the second allele ( $\mathrm{Q} 45 \mathrm{H}, \mathrm{F} 533 \mathrm{Y})$ map to non-conserved regions of the transporters, one 
(F262Y) maps to a site immediately adjacent to the nearly universally-conserved PESPR motif in hexose transporters that is involved in transporter dynamics (Sun et alo, 2012). Furthermore, the mutation from phenylalanine to tyrosine results in an expanded motif (PESPRY) that is present in all of the major hexose transporters in S. cerevisiae (Figure 4B). We then used CRISPRm to introduce the N209S mutation (nucleotide G626A) in the cdt-1045H/F262Y/F533Y diploid yeast background. The quadruple mutant cdt-1 allele resulted in synergistic cellobiose utilization with 3.9-fold increased growth over strains expressing wild-type cdt-1 (Figure 4A). We next tested the selected cdt-1 alleles in anaerobic fermentations using cellobiose as the sole carbon source. We constructed a cdt-1 ${ }^{\text {N2095/F262Y }}$ double-mutant in diploid yeast using CRISPRm, and compared it to wild-type cdt-1 and the cdt-1045H/N2095//262Y/F533Y quadruple mutant. We found that wild-type cdt-1 integrated into the chromosome was barely able to support cellobiose fermentation (Figure 4C). By contrast, the cdt-1045//N2095/F262Y//F533Y quadruple mutant or the cdt-1 ${ }^{\text {N2095/F262Y }}$ double-mutant expressed from the chromosome resulted in complete fermentation of cellobiose, comparable to wild-type cdt-1 expressed from a high-copy $2 \mu$ plasmid (Figure $4 C$; Ha et al., 2011).

\section{Discussion}

We have demonstrated that CRISPRm can be used to quickly generate markerless loss-of-function alleles, heterologous insertion alleles, allele swaps and engineer proteins in yeast by in vivo selections for greatly improved metabolic activity. Although we selected for improved protein function, as suggested by the location of the mutations in CDT-1 (Figure 4B), it is also possible that selection enriched for sequences that confer improved mRNA stability or translation efficiency, due to the fact that the selection was carried out in vivo. Future experiments will be needed to distinguish between these possibilities.

CRISPRm could be used to integrate DNA libraries to interrogate cis-regulatory elements such as transcription promoters, $5^{\prime}$ - and $3^{\prime}$-untranslated regions (5'-UTRs and $3^{\prime}$-UTRs) in mRNAs, or riboswitches. CRISPRm enables these types of experiments because it allows for near quantitative integration of DNA libraries, which can be a single fragment or complex assemblies of multiple fragments. This could enable integration and selection of multi-gene pathways, or specific mutation and selection of subsets of a protein or multiple DNA-encoded functions.

The ability to use chromosomally-integrated DNA libraries removes a key limitation in in vivo selection experiments by eliminating biomolecule expression variation due to variable plasmid copy numbers. Although we used a relatively small library size in the present experiments, even in this small library we observed impressive gains in transporter function. We observed 2-3 mutations per kilobase in the selected transporter genes, resulting in 3-5 amino acid substitutions per transporter. We were then able to combine the mutations in a single transporter with superior fermentation performance. Library sizes introduced by CRISPRm should be scalable to the limits of transformation efficiency, to $10^{7}$ or larger (Kawai et al., 2010). CRISPRm should also enable multiple rounds of selection for directed evolution experiments. We envision that each cycle would involve error-prone PCR of the gene of interest, either from a starting sequence or an enriched and improved cell population, followed by CRISPRm-mediated integration of the amplified gene and selection. This cycle would compare favorably with that required for plasmid-based selections, yet would avoid the problem of plasmid copy number variation. CRISPRm should also be adaptable to screens that rely on sorting large populations of cells, that is by fluorescence-activated cell sorting. We envision that CRISPRm could improve the discovery of human therapeutics by yeast display (Krugel et al., 1988), and could be used to map the importance of protein-protein interactions (Ovchinnikov et al., 2014) via multiplexed library insertion.

To achieve high efficiency multiplexing with CRISPRm, we found it was necessary to modify the sgRNA design by including a 5' HDV ribozyme, and to assay multiple RNA polymerase III promoters for a given strain background. The ease of discovering tRNAs in any genome and the universality of the sgRNA construct means that, with few modifications, CRISPRm can be used directly in non-model fungal hosts, such as pathogens or organisms used in the biotechnology industry. The genetics of many of these organisms have not been studied in any depth due to the technological limitations of available genetic manipulation techniques. For example, industrial $S$. cerevisiae strains are more stress tolerant and produce much higher yields of desired biofuel or renewable chemical end products than laboratory strains (Kerr and Service, 2005; Farrell et al., 2006; Rubin, 2008). However, linking genotypes of industrial yeasts to their phenotypes remains difficult. CRISPRm should serve as a rapid 
and high throughput means for connecting the genotypes of these organisms to their phenotypes, that is for generating marker-free barcoded alleles for large-scale pooled fitness studies of loss-offunction mutants in these organisms (Giaever et alo, 2002). In the future, CRISPRm may also be applicable to interrogating mammalian cells, if the levels of HDR are sufficiently high. Finally, the ability to use CRISPRm for multiplexed targeting paves the way for applying directed evolution to cellular pathways and genetic circuits for higher order synthetic biology applications in any host strain.

\section{Materials and methods}

\section{Cloning the pCAS plasmid backbone}

Gibson Assembly Mastermix (E2611L) (New England Biolabs, Ipswich, MA) (Gibson et alo, 2009) was used to fuse the KANMX (Yeastdeletionpages.com) cassette to the pUC bacterial origin of replication from pESC-URA (Agilent Technologies, Santa Clara, CA). Restriction-free (RF) (van den Ent and Löwe, 2006) cloning was used to add a yeast $2 \mu$ origin of replication from pESC-URA to the pCAS backbone. The resulting pCAS backbone plasmid was propagated in yeast to confirm functionality.

\section{Cas9 expression constructs}

The Cas9 gene from S. pyogenes was amplified from clone MJ824 (Jinek et al., 2012) and cloned into the pCAS backbone plasmid by RF cloning. A yeast nuclear localization signal (NLS) sequence, codon optimized using IDT software (Integrated DNA Technologies, Coralville, IO), was then cloned into the plasmid by RF cloning. Additional elements fused by RF cloning to the Cas9-NLS sequence included the GFP gene, the CYC1 terminator from S. cerevisiae strain S288c (Yeastgenome.org) and the promoters (800 base pairs upstream) from the genes TDH3, TEF1, RNR2 and REV1, also taken from strain S288c (Lee et al., 2013). For genome editing experiments, the GFP sequence was removed from the Cas9 gene and replaced with a C-terminal His ${ }_{8}$ affinity tag, by RF cloning.

\section{Engineering of sgRNA constructs}

Synthetic DNA (Integrated DNA Technologies, Coralville, IO) for the sgRNA and for a catalytically active form of the Hepatitis Delta Virus (HDV) Ribozyme was sequentially cloned by RF cloning into the Cas9 containing vector (pCAS). The terminator (200 bp) of SNR52 (Yeastgenome.org) was cloned 3' of the ribozyme-sgRNA sequence by RF cloning. A series of RNA polymerase III (Pol III) promoters were PCR amplified from S288c genomic DNA and cloned 5' of the ribozyme-sgRNA sequence by RF cloning. The tRNA promoters included the full-length tRNA plus 100 base pairs upstream of the tRNA gene (Supplementary file 1C). The sgRNAs used for multiplex targeting were PCR amplified using primers containing $5^{\prime}$ and $3^{\prime}$ restriction sequences and sub-cloned into pCAS by ligation dependent cloning into Sall, Spel and Sacll unique restriction sites.

\section{Yeast strains used in this study}

S. cerevisiae strain S288c (204508; ATCC) (American Type Culture Collection, Manassas, VA) was used as the haploid, and then mated to form the homozygous diploid for the diploid experiments. Yeast strain ATCC4124 is an industrial polyploid yeast isolated from a molasses distillery, and was obtained from ATCC (American Type Culture Collection, Manassas, VA).

\section{Cas9-GFP localization and expression}

Expression and localization of Cas9-GFP was verified by imaging haploid S. cerevisiae S288c cells transformed with pCas9-GFP::KAN using fluorescence microscopy (Leica Epifluorescence, Leica Microsystems, Buffalo Grove, IL). Cells were grown overnight and nuclear localization visualized at 100× magnification.

\section{Fitness analysis of Cas9 expressed by different promoters}

Yeast cells containing pCAS (Cas9-His 8 variant) were grown in a Bioscreen C Growth Curve Analyzer (Growth Curve USA, Piscataway, NJ) in $200 \mu$ l of YPD + G418 liquid medium (20 g/l Peptone [211667; Bacto], $10 \mathrm{~g} / \mathrm{l}$ Yeast Extract [212750; Bacto], $0.15 \mathrm{~g} / \mathrm{l}$ Adenine hemisulfate [A9126; Sigma] and $20 \mathrm{~g} / \mathrm{l}$ Glucose [G8270; Sigma] + 200 mg/l G418 [29065A; Santa Cruz Biotechnology]). Cells were grown in three biological replicates each with five technical replicates for $48 \mathrm{hr}$ at $30^{\circ} \mathrm{C}$ under constant shaking. The wild-type control containing an empty vector (POR1.1) was also grown in five technical replicates. Mean and standard deviations of the optical density at $600 \mathrm{~nm}$ were calculated for each time point measured by the Bioscreen. 


\section{CRISPR-Cas9 screening protocol}

The Cas9 transformation mix consisted of $90 \mu$ yeast competent cell mix $\left(\mathrm{OD}_{600}=1.0\right), 10.0 \mu \mathrm{l} \times 10 \mathrm{mg} / \mathrm{ml}$ ssDNA (D9156; Sigma, St. Louis, MO), $1.0 \mu \mathrm{g}$ pCAS plasmid, $5.0 \mu \mathrm{g}$ of linear repair DNA and $900 \mu \mathrm{l}$ Polyethyleneglycol ${ }_{2000}$ (295906; Sigma), $0.1 \mathrm{M}$ Lithium acetate (517992; Sigma) $0.05 \mathrm{M}$ Tris- $\mathrm{HCl}$ (155568; Invitrogen) EDTA (10618973; Fisher Scientific). To measure Cas9 independent integration, the linear DNA was co-transformed with a plasmid lacking the Cas9 protein and sgRNA (pOR1.1) (Supplementary file 2B). Cells were incubated $30 \mathrm{~min}$ at $30^{\circ} \mathrm{C}$, and then subjected to heat shock at $42^{\circ} \mathrm{C}$ for $17 \mathrm{~min}$. Following heat shock, cells were re-suspended in $250 \mu \mathrm{YPD}$ at $30^{\circ} \mathrm{C}$ for $2 \mathrm{hr}$ and then the entire contents were plated onto YPD+G418 plates (20 g/l Peptone, $10 \mathrm{~g} / \mathrm{l}$ Yeast Extract, $20 \mathrm{~g} / \mathrm{l}$ Agar, $0.15 \mathrm{~g} / \mathrm{l}$ Adenine hemisulfate, $20 \mathrm{~g} / \mathrm{l}$ Glucose and $\mathrm{G} 418$ at $200 \mathrm{mg} / \mathrm{l})$. Cells were grown for $48 \mathrm{hr}$ at $37^{\circ} \mathrm{C}$, and colonies imaged using the Biorad ChemiDoc Imager (Biorad, Hercules, CA) before replica plating onto phenotype-selective media. The guide sequences in the sgRNAs used for targeting the various loci are shown in Supplementary file 1D. URA3 mutants were selected on $2.0 \mathrm{~g} / \mathrm{l}$ Yeast nitrogen base without amino acids or ammonium sulfate (Y1251; Sigma), $5.0 \mathrm{~g} / \mathrm{l}$ Ammonium sulfate (A4418; Sigma), $1.0 \mathrm{~g} / \mathrm{l} \mathrm{CSM} \mathrm{(4500-012;} \mathrm{MP} \mathrm{Biosciences),} 20 \mathrm{~g} / \mathrm{l}$ Glucose, $20 \mathrm{~g} / \mathrm{l}$ Agar + 5-fluoroorotic acid (1 g/l) (F-230-25; Goldbio); LYP1 mutants were selected on $2.0 \mathrm{~g} / \mathrm{l}$ Yeast nitrogen base without amino acids or ammonium sulfate, $5.0 \mathrm{~g} / \mathrm{l}$ Ammonium sulfate, $1.0 \mathrm{~g} / \mathrm{l} \mathrm{CSM-lysine} \mathrm{(4510-612;} \mathrm{MP} \mathrm{Biosciences),}$ $20 \mathrm{~g} / \mathrm{l} \mathrm{Glucose}, 20 \mathrm{~g} / \mathrm{l}$ Agar + thialysine (100 mg/l) (A2636; Sigma); CAN1 mutants were selected on $2.0 \mathrm{~g} / \mathrm{l}$ Yeast nitrogen base without amino acids or ammonium sulfate, $5.0 \mathrm{~g} / \mathrm{l}$ Ammonium sulfate, $1.0 \mathrm{~g} / \mathrm{l} \mathrm{CSM}$-arginine (4510-112; MP Biosciences), $20 \mathrm{~g} / \mathrm{l}$ Glucose, $20 \mathrm{~g} / \mathrm{l}$ Agar + canavanine sulfate (50 mg/l) (C9758; Sigma); the remaining auxotrophic mutants were selected on $2.0 \mathrm{~g} / \mathrm{l}$ Yeast nitrogen base without amino acids or ammonium sulfate, $5.0 \mathrm{~g} / \mathrm{l}$ Ammonium sulfate, $1.0 \mathrm{~g} / \mathrm{l} \mathrm{CSM}$, $20 \mathrm{~g} / \mathrm{l} \mathrm{Glucose}$, $20 \mathrm{~g} / \mathrm{l} \mathrm{Agar}$; and aerobic respiration deficient mutants (petites) were selected on $20 \mathrm{~g} / \mathrm{l}$ Peptone, $10 \mathrm{~g} / \mathrm{l}$ Yeast Extract, $20 \mathrm{~g} / \mathrm{l}$ Agar, $0.15 \mathrm{~g} / \mathrm{l}$ Adenine hemisulfate, $20 \mathrm{~g} / \mathrm{l}$ Glycerol (G5516; Sigma). Colonies from the YPD+G418 plates were picked and grown overnight in $0.8 \mathrm{ml}$ of YPD. Genomic DNA was extracted from these cultures using the MasterPure Yeast DNA Extraction Kit (MPY80200; Epicentre). PCR confirmation of the 60-mer integration allele was performed using primers flanking the target site. PCR products were purified by Exo-SAP-IT (78201; Affymetrix) and Sanger sequenced at the UC Berkeley, Sequencing Facility (Berkeley, CA) to confirm barcode sequence in the amplicon.

\section{RT-qPCR of sgRNAs}

Cells containing the pCAS plasmid with sgRNA inserts were grown in $900 \mu \mathrm{l}$ of YPD+G418 medium for $24 \mathrm{hr}$ at $30^{\circ} \mathrm{C}$ and $750 \mathrm{rpm}$. Total RNA was extracted from exponentially growing yeast cells using Ambion RNA RiboPure Yeast Kit (AM1926) (Life Technologies, Carlsbad, CA). RT-qPCR was performed on the Applied Biosciences StepOne Real-Time PCR System (Applied Biosystems, Foster City, CA) using the Invitrogen EXPRESS One-Step SYBR GreenER Kit (Life Technologies, Carlsbad, CA). The RT-qPCR expression level data was quantified using the Comparative $C T_{T}\left(\Delta \Delta C_{T}\right)$ method and relative abundance of the sgRNA was normalized to the mRNA transcript UBC6, which was used as the endogenous control (Teste et al., 2009). The primer sequence used for the RT reaction was $5^{\prime}$-AAAAGCACCGACTCGGT-3' and the additional q-PCR primer used was $5^{\prime}$-GTTTTAGAGCTAGAAATAGCAAG-3'. The primers used for the UBC6 endogenous control were (RT) 5'-CATTTCATAAAAAGGCCAACC-3' and (qPCR) 5'-CCTAATGATAGTTCTTCAATGG-3'. DNA sequences for the sgRNAs used to test HDV ribozyme function are shown in Supplementary file $2 \mathrm{C}$ and Supplementary file 2D.

\section{Multiplex genome targeting by Cas9}

Multiplex targeting was performed as described above using pCAS plasmids containing more than one sgRNA expression construct cloned into one of the restriction sites by ligation dependent cloning. Single vs double mutant efficiency was scored relative to the number of colonies present on the YPD+G418 plate. Genomic DNA isolation and PCR of the integration site was performed as described above.

\section{Multiplex in vivo assembly of DNA using Cas9}

Drug resistance cassettes were assembled in vivo from three linear double-stranded DNA fragments PCR amplified from the Ashbya gossypii TEF1 promoter $\left(A g P_{T E F 1}\right)$, the nourseothricin open reading frame $\left(\mathrm{Nat}^{R}\right)$ and $A$. gossypii TEF1 $\left(A g T_{\text {TEF1 }}\right)$ terminator in separate reactions. The primers used to 
amplify the promoter and terminators contained $50 \mathrm{bp}$ of homology to the nourseothricin ORF and $50 \mathrm{bp}$ of homology to the genomic target. Colonies exhibiting drug resistance (nourseothricin $100 \mathrm{mg} / \mathrm{ll}$ (N-500-1; Goldbio) following replica plating were compared to the number of colonies on the YPD+G418 to determine efficiency of multiplex assembly.

\section{Selection of improved variants of cellodextrin transporter CDT1}

To generate CDT1 mutant allele libraries, the GeneMorph II Random Mutagenesis Kit (200550; Aglient) (Agilent Technologies, Santa Clara, CA) was used to amplify the N. crassa cdt-1 open reading frame. The library of cdt-1 mutant alleles was co-transformed with PCR-amplified linear dsDNA fragments encoding the $S C P_{P G K 1}$ promoter and $S C T_{A D H 1}$ terminator into a diploid $S 288 \mathrm{c}$ yeast strain containing a previously-integrated gh1-1 gene. The gh1-1 gene included the $S_{C} P_{\text {TDH3 }}$ promoter, the N. crassa gh 1-1 open reading frame and $S c T_{C Y C 1}$ terminator. The primers used to amplify the promoters and terminators contained $50 \mathrm{bp}$ of homology to either the cdt-1 or gh1-1 ORFs and $50 \mathrm{bp}$ of homology to the respective the genomic targets. $5 \mu \mathrm{g}$ of each of the three PCR products (promoter, open reading frame, terminator) were co-transformed with the PCAS plasmid containing the URA3 guide sequences and screened for G418 resistance as described. Approximately 1600 G418-resistant colonies were pooled and resuspended in minimal cellobiose medium (SCel) $(2.0 \mathrm{~g} / \mathrm{l}$ Yeast nitrogen base without amino acids or ammonium sulfate, $5.0 \mathrm{~g} / \mathrm{l}$ Ammonium sulfate, $1.0 \mathrm{~g} / \mathrm{l} \mathrm{CSM}, 20 \mathrm{~g} / \mathrm{l}$ Cellobiose). Resuspended cells were immediately spread evenly on SCel plates for initial analysis prior to cellobiose selection ( $t=0$ samples). Ten microliters of the resuspended cells were inoculated in $50 \mathrm{ml}$ of SC medium in biological triplicate. Cells were harvested after 3 days and spread onto SCel plates. Cells were grown at $30^{\circ} \mathrm{C}$ for 4 days, and the largest colonies were chosen for further analysis.

\section{Tecan growth analyzer and fitness calculation of cdt-1 alleles}

Cells were grown overnight in $0.5 \mathrm{ml}$ of Synthetic Dextrose (2\%) (SD) in 96 well plates. Cultures were diluted 1:500 in SCel media (4\% cellobiose) and $150 \mu \mathrm{l}$ were grown using the Tecan Sunrise (Tecan Systems Inc., San Jose, CA) in biological triplicate for 3 days at $30^{\circ} \mathrm{C}$. Average and standard deviation was calculated for each biological sample. Relative fitness was calculated by measuring area between the curves $(A B C)$ which normalizes growth to the area under the curve (AUC) for diploid cells lacking cdt-1 ( $\mathrm{ABC}=\mathrm{AUC}$ cdt-1+-AUC cdt-1-1 $)$. Fold cellobiose utilization capacity $=\left(\mathrm{ABC} c d t-1^{5209} /\right.$ $\mathrm{ABC}$ cdt-1).

\section{Generating the double mutant and quadruple cdt-1 alleles}

Integration of the repair oligonucleotide into the chromosomal wild-type cdt-1 gene or cdt-1045H/F262Y/F533Y was performed as described for integrating barcoded DNA. The sgRNA sequence used to target the wild type allele of cdt-1 was cloned into the pCAS vector with the sequence 5'-TGCACTGGC TTCTACAACTG-3'. The repair oligonucleotide used to make the A626G (i.e., N209S) mutation was 5'-CGGCCGCTGCACTGGCTTCTACAGCTGCGGTTGGTTCGGAGGTTCATTCCTGCCGCCTG-3' with 50 more base pairs of homology to cdt-1, on both sides of the above oligonucleotide. Genomic DNA was extracted as described and Sanger sequencing confirmed the incorporation of the A626G mutation. Similarly, the T785A mutation (F262Y) was incorporated into the cdt-1 $1^{\text {N2095 }}$ allele using an sgRNA with guide sequence $5^{\prime}$-CCTCGCTTCCTATTTGCCAA-3', and a repair oligonucleotide with sequence 5'-AGAATCCCCTCGCTACCTATTTGCCAACGGCCGCGACGCTGAGGCTGTTGCCTTTCTTGT-3'. Genomic DNA was extracted as described and Sanger sequencing confirmed the incorporation of the T785A mutation.

\section{Structural analysis of $\mathrm{cdt}^{-1} \mathbf{1}^{\mathrm{N} 2095 / 045 \mathrm{H} / \mathrm{F} 262 \mathrm{Y} / \mathrm{F5} 33 \mathrm{Y}}$}

The sequences of N. crassa CDT-1 (accession XP_963801), E. coli XlyE (accession YP_492174.1), and S. epidermidis GlcP glucose transporter (accession ZP_04818045.1) were aligned with the MUSCLE algorithm (Edgar, 2004). Figures were prepared with the PyMol molecular graphics system (http:// www.pymol.org/).

\section{Imaging of integrated and plasmid based fluorescent proteins}

Strain yML068 was generated by integrating a short DNA 'spacer' sequence and Leu2 into the LEU2 locus of BY4741. Strain yML069 was generated by integrating $P_{T D H 3}-m R u b y 2-T_{T D H 1}$ and Ura3 into the URA3 locus, and integrating $P_{\text {TDH3 }}$-Venus- $T_{\text {TDH1 }}$ and Leu2 into the LEU2 locus of BY4741. Strain $y M L 104$ was generated by integrating $P_{\mathrm{TDH}}-\mathrm{mRuby} 2-T_{\mathrm{TDH}}{ }^{-} P_{\mathrm{TDH}}-\mathrm{Venus}-T_{\mathrm{TDH} 1}$ and Ura3 into the URA3 locus of 
yML068. Plasmid pML1350 is a Ura3 CEN6/ARS4 plasmid with $P_{T D H 3}$-mRuby2- $T_{T D H 1}$. pML1362 is a Leu2 CEN6/ARS4 plasmid with $P_{T D H 3}-$ Venus- $T_{T D H 1}$. pML1177 is a Ura3 CEN6/ARS4 plasmid with $P_{\mathrm{TDH}}$-mRuby2- $T_{\mathrm{TDH} 1}-P_{\mathrm{TDH}}$-Venus- $T_{\mathrm{TDH}}$.

Synthetic defined media lacking leucine and uracil (SD-LU) was made by adding $6.7 \mathrm{~g} / \mathrm{l}$ Difco Yeast Nitrogen Base without amino acids; 2 g/l Drop-out Mix Synthetic Minus Leucine, Uracil without Yeast Nitrogen Base (US Biological); and $20 \mathrm{~g} / \mathrm{l}$ Dextrose to distilled water.

Colonies were picked into SD-LU media and grown to exponential phase at $30^{\circ} \mathrm{C}$. Cultures were concentrated by centrifugation, spotted onto plain glass slides, and examined on a Zeiss Observer D1 microscope using a 100x DIC objective. Images were captured using a Hamamatsu Orca-flash 4.0 (C11440) camera using auto-exposure. Fluorescence images were taken using an X-Cite Series 120 lamp, Zeiss filter set 45 (excitation at 560/40 nm and emission at 630/75 nm) for mRuby2, and Zeiss filter set 46 (excitation at 500/20 nm and emission at 535/30 nm) for Venus. Images were analyzed and composites were created using Fiji (http://fiji.sc).

\section{Illumina sequencing to identify potential off-target mutations}

To rule out that our CRISPR-Cas9/sgRNA system resulted in non-specific genome targeting, we performed whole genome sequencing for URA3 and LYP1 targeted strains and searched for insertion/ deletions (INDELs), single nucleotide polymorphisms (SNPs) and multi-nucleotide polymorphisms (MNPs). We identified 21 sequence variants across the nine URA3 and LYP1 targeted strains (Supplementary file 1B). Whole genome sequencing was performed by the UC Davis Genome Center (Davis, CA) using the Illumina MiSeq platform (Illumina, Hayward, CA) to produce $150 \mathrm{bp}$ paired-end reads. The software package versions used for sequencing data analysis were as follows: BWA (v. 0.7.5ar405), Picard (v. 1.92[1464]), SAMtools (v. 0.1.19-44428cd) and the GATK (2.7-2g6bda569). The S288C reference genome (v. R64-1-1, release date 3 Feb 2011) was obtained from the Saccharomyces Genome Database (yeastgenome.org) and prepared for use in sequencing data analysis with bwa index, CreateSequenceDictionary from Picard, and samtools faidx. Sequencing reads were processed with Scythe $(v$. 0.991) to remove adapter contamination and Sickle (v. 1.210) to trim low quality bases. Processed reads were mapped to the S288C reference genome using bwa mem with the-M option for picard and GATK compatibility. The mapped reads were sorted with SortSam and duplicate reads were marked with MarkDuplicates from Picard. Read alignments were refined by performing local realignment with the RealignerTargetCreator and IndelRealigner walkers from the GATK on all samples collectively.

Variant detection for both SNPs and INDELs was performed with GATK's UnifiedGenotyper, with parameters adjusted for haploid genomes and no downsampling of coverage, for each sample independently. The resulting SNP and INDEL calls were filtered with the VariantFiltration walker from GATK (see header of the VCF file, supplemental VCF file, for details). A custom Perl script (Supplementary file 3) was written to identify all GG dinucleotide sequences in the S288C reference genome, extract every Cas 9 target sequence (i.e., $20 \mathrm{nt}$ sequence corresponding to the 20 nucleotides immediately 5 ' of the 'NGG' PAM site), and obtain the genome coordinates ranging from 30 nucleotides upstream and downstream of the PAM site. Cas9 target sequences were added to VCF files as custom annotations using snpEff (v3.3h), and SnpSift (v3.3h) was used to extract desired fields into tables for analysis with a custom R script (Supplementary file 4). Needleman-Wunsch global alignments between our guide sequences and Cas9 target sequences were performed using the pairwiseAlignment function (Biostrings package, Bioconductor) in $\mathrm{R}$, with a substitution matrix of -1 for mismatches and 2 for matches, produced with the nucleotideSubstitutionMatrix function (Biostrings package, Bioconductor). The probability of there being a better match for the guide sequence to a given Cas9 target sequence was calculated as the frequency of Cas9 target sequences with better alignments to the same guide sequence, amongst 10,000 randomly selected Cas9 target sequences. To compile counts of all variants and various subclasses, a GATKReport was generated from the VCF files with GATK's VariantEval walker, read into R using the 'gsalib' library, and the desired categories were extracted with a custom R script (Supplementary file 4).

Without the repair DNA template required for HDR, the majority of mutations caused by Cas9 are expected to be INDELs, SNPs or MNPs that initiate within the protospacer sequence, which is the 20 nucleotide (nt) sequence $5^{\prime}$ of the PAM (Fu et al., 2013). Therefore, for completeness, we searched for all PAM sites within $30 \mathrm{nt}$ upstream and downstream for each of the 21 variants. We then compared the $20 \mathrm{nt}$ URA3 and LYP1 guide sequences to the putative protospacer sequences within the regions 
flanking each variant. An end-to-end alignment identified 10 or fewer nucleotide matches between the URA3 or LYP1 guide sequences and the variant sequences (Supplementary file 1B). Cas9 requires at least 12 perfect base pair matches within the guide-target sequence (Hsu et al., 2013) so it is highly unlikely that the URA3 and LYP1 guide sequences directed Cas9 to any of these potential protospacers that lie within variant sites. These mutations likely arose as natural variants during the course of the experiment.

As a second method to evaluate the likelihood of off-target mutations, we performed local alignments of our guide sequences to all Cas9 target sequences whose PAM site was within 30 nucleotides upstream and downstream of a detected variant, as well as to 10,000 randomly selected Cas9 target sequences from the genome. Since our guide sequences are expected to have a better match to $13 \%$ or more of all Cas9 target sequences ( 126,000 or more sites) than to the best matching Cas 9 target sequence with a nearby variant, and the number of nucleotide matches in end-to-end alignments is at most 10, we argue that the variants identified in the genomes of URA3- and LYP1-targeted strains are unlikely the result of off-target Cas9 modifications.

\section{Anaerobic fermentations}

Yeast strain colonies were inoculated in $20 \mathrm{ml}$ of oMM (optimized minimal media) (Yuping Lin, personal communication) with $2 \%$ glucose in $50 \mathrm{ml}$ Falcon tubes and grown aerobically at $30^{\circ} \mathrm{C}$ to saturation for $24 \mathrm{hr}$. The oMM contained $1.7 \mathrm{~g} / \mathrm{YNB}$ (Y1251; Sigma, Saint Louis, MO, USA), $2 \times \mathrm{CSM}, 10 \mathrm{~g} / \mathrm{l}\left(\mathrm{NH}_{4}\right)_{2} \mathrm{SO}_{4}$,

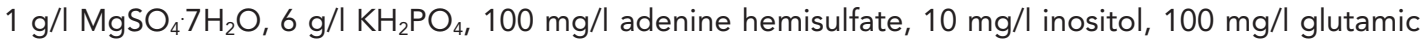
acid, $20 \mathrm{mg} / \mathrm{l}$ lysine, $375 \mathrm{mg} / \mathrm{l}$ serine and $0.1 \mathrm{M}$ 2-( $\mathrm{N}$-morpholino) ethanesulfonic acid (MES) pH 6.0. The saturated cultures were then inoculated to starting OD 600 of 0.2 in $500 \mathrm{ml}$ of oMM with $2 \%$ glucose in 1 I Erlenmeyer flasks and grown aerobically at $30^{\circ} \mathrm{C}$ to mid-log phase at a final OD600 of 2.5. Cells were harvested and washed twice with sterile $\mathrm{dd}_{2} \mathrm{O}$. The washed cells were then inoculated at OD600 of 20 in $50 \mathrm{ml}$ oMM with $1 \%$ glucose and $8 \%$ cellobiose in $125 \mathrm{ml}$ serum flasks. After inoculation, the flasks were sealed with rubber stoppers and clamped with an aluminum seal. To achieve anaerobic conditions, the headspaces of the sealed flasks were purged with nitrogen gas for $30 \mathrm{~min}$. These were then cultivated at $30^{\circ} \mathrm{C}$ and $220 \mathrm{rpm}$.

Using sterile needles and syringes, $1 \mathrm{ml}$ samples were removed through the rubber stoppers at the indicated time points. The cells were pelleted and $5 \mu$ of the supernatants were analyzed for cellobiose, glucose, glycerol, and ethanol content by high performance liquid chromatography on a Prominence HPLC (Shimadzu, Kyoto, Japan) equipped with Rezex RFQ-FastAcid H $10 \times 7.8 \mathrm{~mm}$ column. The column was eluted with $0.01 \mathrm{~N}$ of $\mathrm{H}_{2} \mathrm{SO}_{4}$ at a flow rate of $1 \mathrm{ml} / \mathrm{min}, 55^{\circ} \mathrm{C}$.

\section{Acknowledgements}

We thank D Nunn and J Doudna for helpful discussions. We thank J Waters for assistance in phenotype screening assays. This work was supported by funding from the Energy Biosciences Institute.

\section{Additional information}

Competing interests

OWR: A patent application related to this work has been filed by J Cate and O Ryan on behalf of the Regents of the University of California. JHDC: A patent application related to this work has been filed by $\mathrm{J}$ Cate and $\mathrm{O}$ Ryan on behalf of the Regents of the University of California. The other authors declare that no competing interests exist.

Funding

\begin{tabular}{ll} 
Funder & Author \\
\hline Energy Biosciences Institute & Owen W Ryan, Jeffrey M Skerker, \\
& Matthew J Maurer, Xin Li, Jordan C Tsai, \\
& Snigdha Poddar, Michael E Lee, \\
& Will DeLoache, John E Dueber, \\
& Adam P Arkin, Jamie HD Cate \\
\hline
\end{tabular}

The funder had no role in study design, data collection and interpretation, or the decision to submit the work for publication. 
Author contributions

OWR, JMS, MEL, WDL, Conception and design, Acquisition of data, Analysis and interpretation of data, Drafting or revising the article; MJM, XL, JCT, SP, Acquisition of data, Analysis and interpretation of data, Drafting or revising the article; JED, APA, JHDC, Conception and design, Analysis and interpretation of data, Drafting or revising the article

\section{Additional files}

Supplementary files

- Supplementary file 1. (A) CRISPR-Cas screening results. (B) Potential off-target mutations identified by whole genome sequencing. (C) RNA polymerase III promoter sequences used to express sgRNAs.

(D) Guide sequences used in this study.

DOI: 10.7554/eLife.03703.007

- Supplementary file 2. (A) pCAS-Tyrosine-sgRNA-LYP1. (B) pOR1.1. (C) sgRNA without HDV ribozyme. (D) sgRNA with HDV ribozyme.

DOI: 10.7554/eLife.03703.008

- Supplementary file 3. Custom perl script for Cas9 target identification in S288C genome.

DOI: 10.7554/eLife.03703.009

- Supplementary file 4. Custom R script for yeast genome analysis.

DOI: 10.7554/eLife.03703.010

\section{References}

Boeke JD, LaCroute F, Fink GR. 1984. A positive selection for mutants lacking orotidine-5'-phosphate decarboxylase activity in yeast: 5-fluoro-orotic acid resistance. Molecular \& General Genetics 197:345-346. doi: 10.1007/ BF00330984.

Cong L, Ran FA, Cox D, Lin S, Barretto R, Habib N, Hsu PD, Wu X, Jiang W, Marraffini LA, Zhang F. 2013. Multiplex genome engineering using CRISPR/Cas systems. Science 339:819-823. doi: 10.1126/science.1231143.

DiCarlo JE, Conley AJ, Penttilä M, Jäntti J, Wang HH, Church GM. 2013a. Yeast oligo-mediated genome engineering (YOGE). ACS Synthetic Biology 2:741-749. doi: 10.1021/sb400117c.

DiCarlo JE, Norville JE, Mali P, Rios X, Aach J, Church GM. 2013b. Genome engineering in Saccharomyces cerevisiae using CRISPR-Cas systems. Nucleic Acids Research 41:4336-4343. doi: 10.1093/nar/gkt135.

Edgar RC. 2004. MUSCLE: multiple sequence alignment with high accuracy and high throughput. Nucleic Acids Research 32:1792-1797. doi: 10.1093/nar/gkh340.

Esvelt KM, Carlson JC, Liu DR. 2011. A system for the continuous directed evolution of biomolecules. Nature 472:499-503. doi: 10.1038/nature09929.

Farrell AE, Plevin RJ, Turner BT, Jones AD, O'Hare M, Kammen DM. 2006. Ethanol can contribute to energy and environmental goals. Science 311:506-508. doi: 10.1126/science.1121416.

Fu Y, Foden JA, Khayter C, Maeder ML, Reyon D, Joung JK, Sander JD. 2013. High-frequency off-target mutagenesis induced by CRISPR-Cas nucleases in human cells. Nature Biotechnology 31:822-826. doi: 10.1038/nbt.2623.

Galazka JM, Tian C, Beeson WT, Martinez B, Glass NL, Cate JH. 2010. Cellodextrin transport in yeast for improved biofuel production. Science 330:84-86. doi: 10.1126/science.1192838.

Giaever G, Chu AM, Ni L, Connelly C, Riles L, Véronneau S, Dow S, Lucau-Danila A, Anderson K, André B, Arkin AP, Astromoff A, El-Bakkoury M, Bangham R, Benito R, Brachat S, Campanaro S, Curtiss M, Davis K, Deutschbauer A, Entian KD, Flaherty P, Foury F, Garfinkel DJ, Gerstein M, Gotte D, Güldener U, Hegemann JH, Hempel S, Herman Z, Jaramillo DF, Kelly DE, Kelly SL, Kötter P, LaBonte D, Lamb DC, Lan N, Liang H, Liao H, Liu L, Luo C, Lussier M, Mao R, Menard P, Ooi SL, Revuelta JL, Roberts CJ, Rose M, Ross-Macdonald P, Scherens B, Schimmack G, Shafer B, Shoemaker DD, Sookhai-Mahadeo S, Storms RK, Strathern JN, Valle G, Voet M, Volckaert G, Wang CY, Ward TR, Wilhelmy J, Winzeler EA, Yang Y, Yen G, Youngman E, Yu K, Bussey H, Boeke JD, Snyder M, Philippsen P, Davis RW, Johnston M. 2002. Functional profiling of the Saccharomyces cerevisiae genome. Nature 418:387-391. doi: 10.1038/nature00935.

Gibson DG, Young L, Chuang RY, Venter JC, Hutchison CA III, Smith HO. 2009. Enzymatic assembly of DNA molecules up to several hundred kilobases. Nature Methods 6:343-345. doi: 10.1038/nmeth.1318.

Ha SJ, Galazka JM, Kim SR, Choi JH, Yang X, Seo JH, Glass NL, Cate JH, Jin YS. 2011. Engineered Saccharomyces cerevisiae capable of simultaneous cellobiose and xylose fermentation. Proceedings of the National Academy of Sciences of USA 108:504-509. doi: 10.1073/pnas.1010456108.

Houseley J, Tollervey D. 2009. The many pathways of RNA degradation. Cell 136:763-776. doi: 10.1016/j. cell.2009.01.019.

Hsu PD, Scott DA, Weinstein JA, Ran FA, Konermann S, Agarwala V, Li Y, Fine EJ, Wu X, Shalem O, Cradick TJ, Marraffini LA, Bao G, Zhang F. 2013. DNA targeting specificity of RNA-guided Cas9 nucleases. Nature Biotechnology 31:827-832. doi: 10.1038/nbt.2647. 
Iancu CV, Zamoon J, Woo SB, Aleshin A, Choe JY. 2013. Crystal structure of a glucose/H+ symporter and its mechanism of action. Proceedings of the National Academy of Sciences of USA 110:17862-17867. doi: 10.1073/pnas.1311485110.

Jinek M, Chylinski K, Fonfara I, Hauer M, Doudna JA, Charpentier E. 2012. A programmable dual-RNA-guided DNA endonuclease in adaptive bacterial immunity. Science 337:816-821. doi: 10.1126/science.1225829.

Jinek M, Jiang F, Taylor DW, Sternberg SH, Kaya E, Ma E, Anders C, Hauer M, Zhou K, Lin S, Kaplan M, lavarone AT, Charpentier E, Nogales E, Doudna JA. 2014. Structures of Cas9 endonucleases reveal RNA-mediated conformational activation. Science 343:1247997. doi: 10.1126/science.1247997.

Kawai S, Hashimoto W, Murata K. 2010. Transformation of Saccharomyces cerevisiae and other fungi: methods and possible underlying mechanism. Bioengineered Bugs 1:395-403. doi: 10.4161/bbug.1.6.13257.

Ke A, Ding F, Batchelor JD, Doudna JA. 2007. Structural roles of monovalent cations in the HDV ribozyme. Structure 15:281-287. doi: 10.1016/j.str.2007.01.017.

Kerr RA, Service RF. 2005. What can replace cheap oil-and when? Science 309:101. doi: 10.1126/ science.309.5731.101.

Köhler A, Hurt E. 2007. Exporting RNA from the nucleus to the cytoplasm. Nature Reviews Molecular Cell Biology 8:761-773. doi: 10.1038/nrm2255.

Krugel H, Fiedler G, Haupt I, Sarfert E, Simon H. 1988. Analysis of the nourseothricin-resistance gene (nat) of Streptomyces noursei. Gene 62:209-217. doi: 10.1016/0378-1119(88)90559-8.

Lee ME, Aswani A, Han AS, Tomlin CJ, Dueber JE. 2013. Expression-level optimization of a multi-enzyme pathway in the absence of a high-throughput assay. Nucleic Acids Research 41:10668-10678. doi: 10.1093/nar/gkt809.

Mali P, Esvelt KM, Chruch GM. 2013a. Cas9 as a versatile tool for engineering biology. Nature Methods 10 : 957-963. doi: 10.1038/nmeth.2649.

Mali P, Yang L, Esvelt KM, Aach J, Guell M, DiCarlo JE, Norville JE, Church GM. 2013b. RNA-guided human genome engineering via Cas9. Science 339:823-826. doi: 10.1126/science.1232033.

Marck C, Kachouri-Lafond R, Lafontaine I, Westhof E, Dujon B, Grosjean H. 2006. The RNA polymerase III-dependent family of genes in hemiascomycetes: comparative RNomics, decoding strategies, transcription and evolutionary implications. Nucleic Acids Research 34:1816-1835. doi: 10.1093/nar/gkl085.

Ness F, Lavallée F, Dubourdieu D, Aigle M, Dulau L. 1993. Identification of yeast strains using the polymerase chain reaction. Journal of the Science of Food and Agriculture 62:89-94. doi: 10.1002/jsfa.2740620113.

Ovchinnikov S, Kamisetty H, Baker D. 2014. Robust and accurate prediction of residue-residue interactions across protein interfaces using evolutionary information. eLife 3:e02030. doi: 10.7554/eLife.02030.

Rubin EM. 2008. Genomics of cellulosic biofuels. Nature 454:841-845. doi: 10.1038/nature07190.

Shalem O, Sanjana NE, Hartenian E, Shi X, Scott DA, Mikkelsen TS, Heckl D, Ebert BL, Root DE, Doench JG, Zhang F. 2014. Genome-scale CRISPR-Cas9 knockout screening in human cells. Science 343:84-87. doi: 10.1126/science.1247005.

Sternberg SH, Redding S, Jinek M, Greene EC, Doudna JA. 2014. DNA interrogation by the CRISPR RNAguided endonuclease Cas9. Nature 507:62-67. doi: 10.1038/nature13011.

Storici F, Durham CL, Gordenin DA, Resnick MA. 2003. Chromosomal site-specific double-strand breaks are efficiently targeted for repair by oligonucleotides in yeast. Proceedings of the National Academy of Sciences of USA 100:14994-14999. doi: 10.1073/pnas.2036296100.

Sun L, Zeng X, Yan C, Sun X, Gong X, Rao Y, Yan N. 2012. Crystal structure of a bacterial homologue of glucose transporters GLUT1-4. Nature 490:361-366. doi: 10.1038/nature11524.

Teste MA, Duquenne M, François JM, Parrou JL. 2009. Validation of reference genes for quantitative expression analysis by real-time RT-PCR in Saccharomyces cerevisiae. BMC Molecular Biology 10:99. doi: 10.1186/1471-2199-10-99.

Turner NJ. 2009. Directed evolution drives the next generation of biocatalysts. Nature Chemical Biology 5:567-573. doi: 10.1038/nchembio.203.

van den Ent F, Löwe J. 2006. RF cloning: a restriction-free method for inserting target genes into plasmids. Journal of Biochemical and Biophysical Methods 67:67-74. doi: 10.1016/j.jbbm.2005.12.008.

Webb CH, Riccitelli NJ, Ruminski DJ, Luptak A. 2009. Widespread occurrence of self-cleaving ribozymes. Science 326:953. doi: 10.1126/science.1178084.

Wingler LM, Cornish WW. 2011. Reiterative recombination for the in vivo assembly of libraries of multigene pathways. Proceedings of the National Academy of Sciences of USA 108:15135-15140. doi: 10.1073/pnas.1100507108.

Yokobayashi Y, Weiss R, Arnold FH. 2002. Directed evolution of a genetic circuit. Proceedings of the National Academy of Sciences of USA 99:16587-16591. doi: 10.1073/pnas.252535999.

Zhou H, Cheng JS, Wang BL, Fink GR, Stephanopoulos G. 2012. Xylose isomerase overexpression along with engineering of the pentose phosphate pathway and evolutionary engineering enable rapid xylose utilization and ethanol production by Saccharomyces cerevisiae. Metabolic Engineering 14:611-622. doi: 10.1016/j. ymben.2012.07.011. 